\title{
Presence of paf-acether in rheumatic diseases
}

\author{
P Hilliquin, C J Menkes, S Laoussadi, J Benveniste, B Arnoux
}

\begin{abstract}
Paf-acether (paf) is a naturally occurring phospholipid involved in inflammatory processes. The presence of paf, its precursor lyso paf, and lipo-paf has been determined in blood and synovial fluid from 13 patients with rheumatoid arthritis (RA), 11 with spondylarthropathies, eight with other inflammatory rheumatisms, 13 with chondrocalcinosis, 15 with osteoarthritis, and also in blood from nine healthy subjects. Paf and lipo-paf were measured by rabbit platelet aggregation after isolation by high performance liquid chromatography, whereas lyso paf was first chemically acetylated to give paf. Lipo-paf in blood was higher in patients than in controls; lipo-paf concentrations in blood and in synovial fluid were significantly higher in rheumatoid arthritis than in osteoarthritis and chondrocalcinosis. By contrast, paf and lyso paf reached their lower values in rheumatoid arthritis. The amounts of lipid mediators were not correlated with biological parameters of inflammation. Lipo-paf, which is considered as a storage form of paf, may be the important form of paf in active inflammatory rheumatism.
\end{abstract}

Paf-acether (1-O-alkyl-2-acetyl-sn-glycero-3phosphocholine) (paf) is a phospholipid mediator implicated in various inflammatory diseases. In humans it may be involved in the pathogenesis of asthma, ${ }^{1}$ chronic obstructive pulmonary diseases, and ulcerative colitis. ${ }^{2}$

The discovery of paf in $1972^{3}$ and the elucidation of its structure ${ }^{45}$ was an important addition to the knowledge of prostaglandins and leukotrienes. Several enzymatic steps are involved in the formation of paf, which occurs by cell stimulation. The initial substrate, a membrane phospholipid ether analogue of phosphatidylcholine, is hydrolysed by a phospholipase $\mathrm{A}_{2}$ to form 1-alkyl-2-lysoglycero-phosphocholine (lyso paf-acether). The latter can in turn be acetylated by an acetyltransferase to give paf. Thus activation of phospholipase $A_{2}$ leads to the release of both the precursor of paf and arachidonic acid.

Paf has been obtained in humans from platelets, blood monocytes, neutrophils, and endothelial cells. ${ }^{1}$ Several pathobiological effects of paf have now been recognised. Paf is the most potent platelet aggregating agent described so far. ${ }^{6}$ It is also a neutrophil activating factor, causing chemotaxis and the release of lysosomal enzymes and free radicals. ${ }^{7}$ It has been shown to cause the release of leukotrienes and prostaglandins from various types of cells and organs. It can induce the secretion of interleukin 1 by muramyl dipeptide stimulated human monocytes. ${ }^{8}$ In ovalbumin induced arthritis in the rabbit, paf and its precursor lyso paf have been detected in synovial fluid in the early stages of arthritis; phospholipids were not found in the chronic stages of the disease. ${ }^{9}$

The aim of this study was to evaluate the presence of paf, its precursor lyso paf and paf bound to lipoprotein ${ }^{10}$ in blood samples and synovial fluids from adults with rheumatic diseases. Patients with inflammatory diseases (rheumatoid arthritis, spondylarthropathies) were compared with patients with noninflammatory arthropathies (chondrocalcinosis, osteoarthritis).

\section{Patients and methods}

Sixty patients who have been admitted to hospital were included in this study. Synovial fluid was collected during routine knee arthrocentesis. Blood and synovial fluid samples were obtained from the following groups: patients with rheumatoid arthritis defined according to the American Rheumatism Association criteria ${ }^{11}$, $n=13$, all presenting with active disease with an inflammatory synovial fluid (more than $2 \times 10^{9}$ cells/1); patients with spondylarthropathies, $n=11$, six with a psoriatic arthropathy and five an ankylosing spondylitis; other inflammatory rheumatisms, $n=8$; chondrocalcinosis, $n=13$, all with non-inflammatory synovial fluid; and osteoarthritis, $n=15$, all with non-inflammatory synovial fluid. Blood samples were also collected from nine healthy volunteers. The clinical history and treatment given were known for every patient. Twelve patients with rheumatoid arthritis were treated with a disease modifying drug (hydroxychloroquine, gold salts, D-penicillamine or immunosuppressive treatment). Most of the patients received nonsteroidal anti-inflammatory drugs and/or low dose glucocorticoids.

\section{LABORATORY STUDIES}

Blood and synovial fluid cell counts and the erythrocyte sedimentation rate were determined by routine analysis. Latex and Waaler-Rose tests and antinuclear antibodies were assessed in the patients with inflammatory disease. Blood or synovial fluid samples (one volume) were immediately mixed with $100 \%$ ethanol (four volumes) and stored at room temperature for one hour. Samples were stored at $-20^{\circ} \mathrm{C}$ until assayed. Paf and paf bound to lipoprotein (lipopaf) were determined in blood and in synovial 
fluid samples, whereas lyso paf was only determined in synovial fluid.

DETECTION OF PAF AND DERIVATIVES

Blood and synovial fluid samples were centrifuged at $3000 \mathrm{~g}$ for $10 \mathrm{~min}$ and the supernatants were evaporated. Paf and lipo-paf were characterised by their retention time during high performance liquid chromatography eluted at a flow of $1 \mathrm{ml} / \mathrm{min}$ using a Microporasil silica column with dichloromethane/methanol/water $(60: 50: 5, \mathrm{v} / \mathrm{v})$ as the solvent. ${ }^{12}$ The retention times were 10 and 22 minutes for lipo-paf and paf, respectively.

Paf and lipo paf were assessed by their aggregating activity on washed rabbit platelets as described previously. ${ }^{3}$ Aggregation was performed at $37^{\circ} \mathrm{C}$ with stirring with aspirin treated $(0.1 \mathrm{mmol} / 1$ for $15 \mathrm{~min})$ platelets in the presence of the creatine phosphate/creatine phosphokinase complex ( $1 \mathrm{mM} 10 \mathrm{U} / \mathrm{ml}$ ) to avoid aggregation due to arachidonic acid and 5'-pyrophosphate (ADP).

Paf-acether was also characterised by its sensitivity to $100 \mu \mathrm{g} / \mathrm{ml}$ phospholipase $A_{2}$ from hog pancreas in $10 \mathrm{mM}$ TRIS buffered saline (pH 8.0) containing $10 \mathrm{mmol} \mathrm{Ca}^{2+}$, and the lack of effect of lipase $A_{1}$ from Rhizopus arrhizus in $0.1 \mathrm{M}$ borate buffer (pH 6.5) with $10 \mathrm{M}$ $\mathrm{Ca}^{2+13}$

Paf-acether activity in the sample was measured over the linear portion of a calibration graph obtained daily with synthetic paf-acether. Results are expressed as paf equivalent in $\mathrm{ng} / \mathrm{ml}$ of biological fluid.

Before being measured by platelet aggregation as paf acether, lyso paf was treated by chemical acetylation to give paf. The ethanolic extracts were first evaporated to dryness and treated overnight at room temperature with $200 \mu \mathrm{l}$ dry pyridine and $200 \mu \mathrm{l}$ acetic anhydride. ${ }^{14}$ After the addition of dichloromethane and evaporation of the reagents under a stream of air, the dry residue was dissolved in saline containing $0.25 \%$ bovine serum albumin ( $\mathrm{pH} 7 \cdot 4$ ) and paf was assayed by its aggregating activity as described previously. The amount of lyso paf was calculated as the difference between the amounts of paf measured after and before acetylation of the samples.

STATISTICAL ANALYSIS

Results are expressed as mean (SD). Data were analysed by one way analysis of variance and by Student's $t$ test when appropriate. Correlations were established between quantitative variables.

\section{Results}

The three compounds were detected in samples from all patients, blood and/or synovial fluid, whereas paf was not detected in control subjects. Lipo-paf was significantly higher $(p<0.05)$ in blood samples from patients than from controls $4 \cdot 1(4 \cdot 2) v 0 \cdot 9(1 \cdot 2) \mathrm{ng} / \mathrm{ml}$ ) (table).

Statistically significant differences were seen in the amount of lipo-paf in blood samples and in synovial fluid from subjects with rheumatoid arthritis and the non-inflammatory groups (table). In rheumatoid arthritis, lipo-paf was significantly higher than in chondrocalcinosis (blood, $\mathrm{p}<0.01$; synovial fluid, $\mathrm{p}<0.01$ ) and osteoarthritis (blood, $\mathrm{p}<0.02$; synovial fluid, $\mathrm{p}<0.03$ ). Lipo-paf in blood samples was also higher in patients with rheumatoid arthritis than in those with spondylarthropathies $(\mathrm{p}<0.02)$. Such differences were not observed between patients with chondrocalcinosis and those with osteoarthritis. There was no significant difference for lipo-paf in blood samples or in synovial fluid for patients with rheumatoid arthritis or spondylarthropathies compared with the other inflammatory rheumatisms, probably because of the lower number of patients in the latter group. In summary, lipo-paf reached its highest values in blood samples and synovial fluid from patients with rheumatoid arthritis, a disease showing a local inflammatory process.

Similar differences between groups were not observed for paf levels in blood samples and in synovial fluid and for lyso paf in synovial fluid. In contrast, paf and lyso paf reached their lowest values in patients with rheumatoid arthritis (table). The differences in paf articular values reached significance when comparing patients with chondrocalcinosis with those with rheumatoid arthritis $(\mathrm{p}<0.02)$. The same pattern was observed for synovial lyso paf between patients with rheumatoid arthritis and those with chondrocalcinosis $(p<0.03)$. Lyso paf in synovial fluid was also significantly higher in patients with spondylarthropathies than in those with rheumatoid arthritis $(\mathrm{p}<0.01)$. Paf and lyso paf values were not significantly different between patients with rheumatoid arthritis and osteoarthritis.

Paf and its derivatives were not significantly correlated with the leucocyte count in blood

Presence of lipo-paf, paf and lyso paf in patients with rheumatic diseases

\begin{tabular}{|c|c|c|c|c|c|}
\hline \multirow[t]{2}{*}{ Subjects } & \multicolumn{2}{|l|}{$\begin{array}{l}\text { Lipo paf } \\
(n g / m l)\end{array}$} & \multicolumn{2}{|l|}{$P a f$} & \multirow{2}{*}{$\begin{array}{l}\text { Lyso paf } \\
\text { Synovial } \\
\text { fluids }\end{array}$} \\
\hline & Blood ${ }^{*}$ & $\begin{array}{l}\text { Synovial } \\
\text { fluidt }\end{array}$ & Blood & $\begin{array}{l}\text { Synovial } \\
\text { fluid } \neq\end{array}$ & \\
\hline $\begin{array}{l}\text { Controls }(n=9) \\
\text { Rheumatoid arthritis }(n=13) \\
\text { Spondylarthropathies }(n=11) \\
\text { Inflammatory rheumatisms }(n=8) \\
\text { Chondrocalcinosis }(n=13) \\
\text { Osteoarthritis }(n=15)\end{array}$ & $\begin{array}{l}0 \cdot 9(1 \cdot 2) \\
9 \cdot 7(6 \cdot 6) \\
2 \cdot 1(1 \cdot 7) \\
1 \cdot 8(1 \cdot 4) \\
2 \cdot 2(1 \cdot 8) \\
3 \cdot 4(3 \cdot 2)\end{array}$ & $\begin{array}{l}\text { NA } \\
6 \cdot 4(5 \cdot 7) \\
3 \cdot 6(2 \cdot 0) \\
3 \cdot 8(1 \cdot 9) \\
1 \cdot 2(1 \cdot 3) \\
2 \cdot 6(2 \cdot 4)\end{array}$ & $\begin{array}{l}0.00(0.00) \\
0.09(0.14) \\
0.65(0.82) \\
0.43(0.72) \\
0.20(0.27) \\
0.10(0.21)\end{array}$ & $\begin{array}{l}\text { NA } \\
0 \cdot 11(0.33) \\
0 \cdot 19(0.40) \\
0 \cdot 14(0.35) \\
0.39(0.77) \\
0.39(1 \cdot 15)\end{array}$ & $\begin{array}{l}\text { NA } \\
1292(549) \\
2700(1164) \\
2001(1201) \\
2446(1726) \\
1844(1662)\end{array}$ \\
\hline
\end{tabular}

Results are expressed as mean (SD). NA=not applicable.

${ }^{*}$ Controls $v$ all patients, $\mathrm{p}<0.05$. Patients with rheumatoid arthritis $v$ spondylarthropathies, $\mathrm{p}<0.02$; rheumatoid arthritis $v$ chondrocalcinosis, $\mathrm{p}<0.01$; rheumatoid arthritis $v$ osteoarthritis, $\mathrm{p}<0.02$.

Patients with rheumatoid arthritis $v$ chondrocalcinosis, $\mathbf{p}<0.01$; rheumatoid arthritis $v$ osteoarthritis, $\mathbf{p}<0.03$.

$\ddagger$ Patients with rheumatoid arthritis $v$ chondrocalcinosis, $\mathrm{p}<0.02$.

SPatients with rheumatoid arthritis $v$ spondylarthropathies, $\mathrm{p}<0 \cdot 01$; rheumatoid arthritis $v$ chondrocalcinosis, $\mathrm{p}<0.03$. 
samples and synovial fluid; paf, lyso paf and lipo paf levels were not correlated with biological parameters of disease activity in patients with inflammatory disease (haemoglobin, erythrocyte sedimentation rate, latex and Waaler-Rose tests, antinuclear antibodies). Neither could we show significant correlations between paf and its derivatives in synovial fluid $v$ blood samples; the only observed correlation was between lipopaf in blood and in synovial fluid in patients with osteoarthritis $(p<0.05)$. The amounts of paf, lyso paf and lipo-paf did not correlate with treatment. However, in patients with osteoarthritis, there was a striking correlation between paf and lyso paf in synovial fluid; in synovial fluid from patients with rheumatoid arthritis, paf was strongly correlated only with lipo-paf, supporting a role for this latter form of the mediator in the active inflammatory disease.

\section{Discussion}

Until now, several mediators have been implicated in the pathogenesis of chronic joint inflammation. LTB4 was found in high amounts in synovial fluid from patients with rheumatoid arthritis ${ }^{15}$ and interleukin 1 has been shown to activate synovial cells. ${ }^{16}$ However, nothing was known about the presence of paf, lyso paf and lipo-paf in blood samples and synovial fluid from patients with rheumatic diseases. Paf and its precursor lyso paf were detected in synovial fluid in the acute stage of an ovalbumin induced arthritis in rabbits, whereas paf and its derivatives were not present in chronic stages of the disease. ${ }^{9}$ The data presented here indicate that paf, lyso paf, and lipo-paf are present in blood and synovial fluid from patients with rheumatic diseases. Furthermore lipo-paf seems to be the main form of the mediator in active inflammatory diseases.

The highest values for lipo-paf were found in patients with rheumatoid arthritis; patients with chondrocalcinosis or osteoarthritis had a significantly lower concentration of lipo-paf than patients with rheumatoid arthritis, either in blood samples or synovial fluid. There was no difference for lipo-paf between patients with spondylarthropathies and the non-inflammatory groups. Paf and lyso paf seemed to vary in the opposite direction to lipo-paf, showing lower values in patients with rheumatoid arthritis. However, this difference reached significance only when comparing the patients with rheumatoid arthritis and those with chondrocalcinosis. A correct interpretation of these data is not obvious; paf and lyso paf could be metabolised very quickly in cases with an active local inflammatory process. A greater number of patients must be studied to determine whether there is a real difference in paf and lyso paf values between patients with rheumatoid arthritis and those with non-inflammatory disease. Paf levels were not correlated with the consumption of disease modifying drugs, glucocorticoids or non-steroidal anti-inflammatory drugs. There was no correlation between paf and its derivatives and the biological parameters of inflammation. Information is lacking on the origin of the paf and lipo-paf detected in synovial fluid. However, most cells present in the synovial pannus (polymorphonuclear cells, monocytes, endothelial cells), with the exception of lymphocytes, are putatively able to synthetise paf, supporting a local synthesis of the mediators. Nevertheless, a passive transfer from blood to the joint space cannot be excluded. Paf could also attract, by its chemotactic action, inflammatory cells into the joint, thus enhancing the local production of paf and its derivatives.

These results show that lipo-paf, which could represent a storage form of paf, is increased in the blood and synovial fluid of patients with inflammatory rheumatic diseases, especially those with rheumatoid arthritis, in agreement with the systemic nature of these diseases. Its value as a putative pathogenic index of chronic joint inflammation, either by itself or by interaction with cytokines, remains to be evaluated. With the ongoing development of paf inhibitors, it is useful to know that paf can be detected in patients with inflammatory rheumatic diseases.

P Hilliquin was supported by a grant from the Fondation de la Recherche Médicale. The authors thank A Landes for her technical assistance.

1 Benveniste J, Arnoux B. Proceeding of the first international symposium on platelet-activating factor and structurally related ether-lipids. INSERM Symposium 23. Amsterdam: Elsevier, 1983.

2 Stenson $W$ F. Platelet-activating factor and inflammatory bowel disease. Gastroenterology 1988; 95: 1416-9.

3 Benveniste J, Henson P M, Cochrane C G. Leukocyte dependent histamine release from rabbit platelets. The role of IgE, basophils, and a platelet-activating factor. f Exp Med 1972; 136: 1357-77.

4 Benveniste J, Tence M, Varenne P, Bidault J, Boullet C, Polonsky J. Semi-synthèse et structure proposée du facteur activant les plaquettes (P.A.F.): paf-acether, un alkyl-éther analogue de la lysophosphatidylcholine. Compte Rendu of the Academy of Sciences (Paris) Série D, 1979; 289: 1037-40.

5 Demopoulos C A, Pinckard R N, Hanahan D J. Plateletactivating factor. Evidence for 1-O-alkyl-2-acetyl-snglyceryl-3-phosphorylcholine as the active component (a new class of lipid chemical mediators). $\mathcal{f}$ Biol Chem 1979; 254: 9355-8.

6 Benveniste J, Le Couedic J P, Kamoun P. Aggregation of human platelets by platelet-activating factor. Lancet 1975 ; i: 344.

7 Czarnetzki M B, Benveniste J. Effect of 1-O-alkyl-2-acetylsn-glyceryl-3-phosphorylcholine (paf-acether) on leukocytes. I. Analysis of the in vitro migration of human neutrophils. Chem Phys Lipids 1981; 29: 317-26.

8 Salem P, Deryckx S, Dulicust A, et al. Paf-acether enhances interleukin-1 secretion by muramyl dipeptide-stimulated human monocytes. F Immunol 1990; 144: 1338-44.

9 Pettipher E R, Higgs G A, Henderson B. Paf-acether in chronic arthritis. Agents Actions 1987; 21: 97-103.

10 Benveniste J, Nunez D, Duriez P, Korth R, Bidault J, Fruchart J C. Preformed PAF-acether and lyso PAF226: 371-6.

1 Arnett F C, Edworthy S M, Bloch D A, et al. The American Rheumatism Association 1987 revised criteria for the classification of rheumatoid arthritis. Arthritis Rheum 1988; 31: 315-24.

12 Tence M, Polonsky J, Le Couedic J P, Benveniste J. Release, purification and characterisation of platelet-activating factor (PAF). Biochimie 1980; 62: 251-9.

13 Benveniste J, Le Couedic J P, Polonsky J, Tence $M$. Structural analysis of purified platelet-activating factor by lipases. Nature 1977; 269: 170.

14 Polonsky J, Tence M, Varenne P, Das B C, Lunel J, Benveniste J. Release of 1-O-alkyl-glyceryl-3-phosphorylcholine, $O$-deacetyl platelet-activating factor, from leukocytes: chemical ionization mass spectrometry of phospholipids. Proc Natl Acad Sci USA 1980; 77: 7019-23.

15 Henderson B, Pettipher E R, Higgs G A. Mediators of rheumatoid arthritis. Br Med Bull 1987; 43: 415-28.

16 Dayer J M, Rochemonteix B, Burrus B, Demczuk S, Dinarello C A. Human recombinant interleukin-1 stimulates collagenase and prostaglandin E2 production by human synovial cells. $\mathcal{f}$ Clin Invest 1986; 77: 645-8. 